\title{
PRIMEIRO RELATO DE OCORRÊNCIA DE Anastrepha serpentina E Anastrepha leptozona (DIP.: TEPHRITIDAE) EM ABIU (Pouteria caimito) NO ESTADO DE SÃO PAULO ${ }^{1}$
}

\author{
ADALTON RAGA²; ROGÉRIO AMARO MACHADO ${ }^{3}$; ANTONIO ALBERTO COSTA ${ }^{4}$; MIGUEL FRANCISCO DE SOUZA \\ FILHO $^{2}$; RENATO FERRAZ DE ARRUDA VEIGA ${ }^{4}$; LUIS ALBERTO SAES ${ }^{5}$
}

RESUMO - Foi registrada pela primeira vez a infestação das moscas-das-frutas Anastrepha serpentina (Wied.) e Anastrepha leptozona Hendel (Dip.: Tephritidae) em abiu Pouteria caimito (Sapotaceae) no Estado de São Paulo. Os frutos coletados foram oriundos da Coleção de Fruteiras Nativas e Exóticas, localizada na sede do Pólo Regional de Desenvolvimento Tecnológico do Vale do Ribeira (SP), município de Pariquera-Açu. Também emergiram espécimens de Neosilba glaberrima (Wied.) (Lonchaeidae).

Termos para indexação: Insecta, Ecologia, Hospedeiro, Sapotaceae

\section{FIRST OCCURRENCE OF Anastrepha serpentina AND Anastrepha leptozona (DIP.: TEPHRITIDAE) ON ABIU (Pouteria caimito) IN THE SATE OF SÃO PAULO, BRAZIL}

ABSTRACT - It was registered for the first time the occurrence of fruit flies Anastrepha serpentina (Wied.) and Anastrepha leptozona Hendel (Dip.: Tephritidae) on abiu, Pouteria caimito (Sapotaceae) in the State of São Paulo, Brazil. Infested fruits were picked up from the Collection of Exotic and Native Tropical Fruits, installed in Pariquera-Açú County. Also, it was found emerged specimens of Neosilba glaberrima (Wied.) (Lonchaeidae) in the same field.

Index terms: Insecta, Ecology, Host Plant, Sapotaceae

O abieiro, Pouteria caimito (Ruiz \& Pav.) Radlk., é uma planta da Família Sapotaceae, considerada originária da região amazônica, nos limites do Brasil, Colômbia, Peru e Venezuela (Manica, 2000). Embora pouco explorado comercialmente, o abiu é uma fruta bastante conhecida nos trópicos, ao lado de outras sapotáceas, como o sapoti (Manilkara zapota L. von Royen), o canistel [Pouteria campechiana (Kunth) Baehni], o caimito (Chrysophyllum cainito L.) e o mamey [Pouteria sapota (Jacq.) Moore \& Stearn] (Lederman et al., 2001).

Dentre as pragas infestantes de sapotáceas em várias regiões tropicais, são encontrados os insetos popularmente conhecidos como 'moscas-das-frutas' (Diptera: Tephritidae). Anastrepha barnesi Aldrich, A. benjamini Lima, A. bistrigata Bezzi, A. bondari Lima, A. fraterculus (Wied.), A. leptozona Hendel, A. macrura Hendel, A. serpentina (Wied.), A. submunda Lima e A. zernyi Lima atacam plantas da família Sapotaceae no Brasil (Zucchi 2000a), além de Ceratitis capitata (Wied.) (Zucchi, 2000b).

No Estado de São Paulo, A. fraterculus foi observada em frutos de abiu (Arrigoni, 1984; Souza Filho, 1999) e caimito mexicano (Pouteria mexicanum Brandegee) (Souza Filho, 1999); A. barnesi em Pouteria torta (Mart.) Radlk (Malavasi et al., 1980); A. serpentina em sapoti (Malavasi et al., 1980), caimito e caimito mexicano (Souza Filho, 1999); $C$. capitata em abiu, caimito e caimito mexicano (Arrigoni 1984; Souza Filho, 1999).

Foram coletados ao acaso 28 frutos maduros num total de 1,78 kg, caídos no chão, em três plantas de abiu localizadas na sede do Pólo Regional de Desenvolvimento Tecnológico do Vale do Ribeira (SP), no km 460 da Rodovia Régis Bittencourt, município de Pariquera-Açu ( $\left.24^{\circ} 37^{\prime} \mathrm{S} ; 4^{\circ} 7^{\circ} 52^{\prime} \mathrm{W} ; 26 \mathrm{~m}\right)$. A coleta foi realizada no mês de maio de 2002 , sendo as árvores oriundas de sementes, introduzidas há aproximadamente 30-35 anos pelo Dr. Hermes M. Souza, constituintes da Coleção de Fruteiras Nativas e Exóticas daquela unidade experimental.

A amostra foi conduzida ao Laboratório de Entomologia Econômica do Centro de Pesquisa e Desenvolvimento em Sanidade Vegetal do Instituto Biológico, em Campinas (SP). Os frutos foram acondicionados em caixa plástica com $37 \times 30 \times 14 \mathrm{~cm}$, contendo uma camada de aproximadamente $4 \mathrm{~cm}$ de uma mistura 1:1 de areia e vermiculita. A caixa foi coberta com tecido de algodão e mantida em ambiente de $25^{\circ} \pm 2^{\circ} \mathrm{Ce}$
$65 \pm 10 \%$ de umidade relativa. Após vinte e um dias da coleta, o substrato foi peneirado e os pupários de Tephritoidea foram transferidos para uma gaiola de vidro com volume de $6.000 \mathrm{~cm}^{3}$, para propiciar a emergência dos adultos. Na gaiola de emergência, estavam disponíveis aos adultos água e açúcar + proteína (3:1).

Foram obtidos 161 pupários de Tephritoidea, correspondendo a 5,7 pupários/fruto e 90,4 pupários/kg de fruto. Emergiram 89 adultos de Tephritoidea, sendo 79 espécimens de Anastrepha, dos quais 32 exemplares fêmeas. Deste total, 23 corresponderam a A. serpentina e 9 a $A$. leptozona. Também emergiram 10 adultos de Neosilba glaberrima (Wied.).

Anastrepha serpentina foi observada em abiu pela primeira vez no Brasil através de material coletado em 1913, no Estado da Bahia (Tavares, 1915); tendo sido também registrada em sapoti, abricó Mimusops coriacea (DC.) Miq. (in Mart.), abricó-do-Pará Mamea americana L. (Costa Lima, 1926), massaranduba Mimusops sp. (Costa Lima, 1934) e bacumuchá Pouteria sp. (Bondar, 1949). Além do Brasil, A. serpentina foi registrada na Argentina, Colômbia, Equador, Guiana, Peru, Suriname, Venezuela, Estados Unidos (Texas), México, Guatemala, Panamá, Costa Rica e Ilhas Ocidentais (White \& Elson-Harris, 1994).

Além de Sapotaceae, A. leptozona infesta frutos de Anacardiaceae, Icacinaceae e Myrtaceae (Zucchi, 2000a, Silva \& RonchiTeles, 2000). No Brasil, essa espécie de moscas-das-frutas infesta os seguintes frutos: abiu e bacumuchá (Costa Lima, 1937), canistel, guapeva $P$. gardneriana Radlk. e curriola $P$. ramiflora (Veloso, 1997), caju Anacardium occidentale L. e mari Poraqueiba paraensis Ducke (Silva, 1993). Anastrepha leptozona ocorre também na Bolívia, Guiana , Venezuela, México, Guatemala e Panamá (White \& Elson-Harris, 1994).

Com relação às moscas da família Lonchaeidae, existe controvérsia na literatura quanto ao status de praga (Del Vecchio, 1981). Esse grupo não teria a importância de tefritídeos como A. fraterculus e $C$. capitata, desenvolvendo-se em frutos previamente infestados por estas espécies, conforme afirmou Costa Lima (1926). No entanto, estudos mais recentes têm demonstrado que várias espécies de Neosilba e Dasiops são limitantes à produção de fruteiras comerciais. Trabalhos conduzidos por Souza Filho (1999) e Uchôa-Fernandes (2002), conduzidos nos Estados de São Paulo e Mato Grosso do Sul, concluíram que os

\footnotetext{
(Trabalho 152/2002). Recebido: 15/09/2002. Aceito para publicação: 30/07/2003.

2 Engenheiro Agrônomo, Pesquisador Científico, Instituto Biológico, Caixa Postal 70, 13001-970, Campinas-SP, Brasil. adalton@ biologico.sp.gov.br.

${ }^{3}$ Estudante de Ciências Biológicas da PUC/Campinas. Bolsista do CNPq.

${ }^{4}$ Engenheiro Agrônomo, Pesquisador Científico, Instituto Agronômico, CP 28, 13075-360, Campinas-SP.

Engenheiro Agrônomo, Pesquisador Científico, Pólo Regional de Desenvolvimento Tecnológico dos Agronegócios do Vale do Ribeira, Rodovia Regis Bittencourt Km 460, Pariquera-Açu-SP.
} 
lonqueídeos representaram $11,6 \%$ e $49,1 \%$ dos exemplares de Tephritoidea, respectivamente. Espécimens de Neosilba obtidos de frutos cítricos do Estado de São Paulo corresponderam a 21,0\% do total de Tephritoidea avaliados por Raga et al. (2000).

Neosilba é quase inteiramente restrita à Região Neotropical, sendo que poucas espécies ocorrem na Flórida e sul dos Estados Unidos. Neosilba glaberrima parece ser uma das espécies com maior distribuição e polifagia, ocorrendo nas Ilhas do Caribe, América Central até Peru e sudeste do Brasil (McAlpine \& Steyskal, 1982).

\section{AGRADECIMENTO}

Ao Sr. Pedro Carlos Strix e ao Prof.Dr. Ângelo Pires do Prado da Universidade Estadual de Campinas, pela identificação dos exemplares de Lonchaeidae.

\section{REFERÊNCIASBIBLIOGRÁFICAS}

ARRIGONI, E.B. Dinâmica populacional de moscas-das-frutas (DipteraTephritidae) em três regiões do Estado de São Paulo. 1984. 162f. Tese (Doutorado em Entomologia) - Escola Superior de Agricultura Luiz de Queiroz, Universidade de São Paulo, Piracicaba, 1984.

BONDAR, G. Moscas-das-frutas na Bahia. Bahia Rural, v. 17, n.9, p. 26-27, 1949.

COSTA LIMA, A. Sobre as moscas-das-frutas que vivem no Brasil. Chácaras e Quintais, São Paulo, v. 34, n. 1, p. 21-234, 1926.

COSTA LIMA, A. Moscas-de-frutas do gênero Anastrepha Schiner, 1868. Memórias do Instituto Oswaldo Cruz, Rio de Janeiro, v. 28, n. 4, p. 487-575, 1934.

COSTA LIMA, A. Moscas-de-frutas do gênero Anastrepha (Diptera: Trypetidae). O Campo, Rio de Janeiro, v. 8, n. 90, p. 34-38, 1937.

DEL VECCHIO, M.C. Familia Lonchaeidae (Diptera: Acalyptratae): ocorrência de espécies e respectivos hospedeiros em algumas localidades do Estado de São Paulo. 1991. 58f. Dissertação (Mestrado em Biologia) Universidade Estadual de Campinas, Campinas, 1991.

LEDERMAN, I.E.; SILVA JUNIOR, J.F.; BEZERRA, J.E.F; MOURA, R.J.M. Sapotizeiro (Manilkara zapota L. von Royen). Jaboticabal: Sociedade Brasileira de Fruticultura, 2001.71p.

MALAVASI, A.; MORGANTE, J.S.; ZUCCHI, R.A. Biologia de "moscas-das-frutas" (Diptera: Tephritidae). I. Lista de hospedeiros e ocorrência. Revista Brasileira de Biologia, Rio de Janeiro, v. 40, n.1, p. 9-16, 1980.

MANICA, I. Frutas nativas, silvestres e exóticas 1. Porto Alegre: Cinco Continentes Editora, 2000. 327p.
McALPINE, J.F.; STEYSKAL, G.C. A revision of Neosilba McAlpine with a key to the world genera of Lonchaeidae (Diptera). The canadian entomologist, Ottawa, v. 114, n.2, p. 105-137, 1982.

SILVA, N.M. Levantamento e análise faunística de moscas-das-frutas (Diptera: Tephritidae) e seus parasitóides (Hymenoptera) em quatro locais do estado do Amazonas. 1993. 152f. Tese (Doutorado em Entomologia) - Escola Superior de Agricultura "Luiz de Queiroz", Universidade de São Paulo, Piracicaba, 1993.

SILVA, N.M.; RONCHI-TELES, B. Moscas-das-frutas nos Estados brasileiros: Amapá, Amazonas, Pará, Rondônia e Roraima. In: Malavasi, A. e Zucchi, R.A. [Eds.]. Moscas-das-frutas de Importância Econômica no Brasil - conhecimento básico e aplicado. Ribeirão Preto: Holos Editora, 2000. p. 203-209.

RAGA, A., PRESTES, D.A.O., SOUZA FILHO, M.F., SATO, M.E., SILOTO, R.C. Fruit fly infestation in citrus cultivated in the State of São Paulo, Brazil. In: INTERNATIONAL SOCIETY OF CITRICULTURE CONGRESS, 9., 2000, Orlando. Abstracts... p. 103.

SOUZA FILHO, M.F. Biodiversidade de moscas-das-frutas (Diptera: Tephritidae) e seus parasitóides (Hymenoptera) em plantas hospedeiras no Estado de São Paulo. 1999. 173f. Dissertação (Mestrado em Entomologia) - Escola Superior de Agricultura "Luiz de Queiroz", Universidade de São Paulo, Piracicaba, 1999.

TAVARES, J.S. A Anastrepha serpentina Wiedm. Nova praga dos frutos no Brasil. Broteria, série zoológica, Lisboa, v. 13, p. 52-54, 1915.

UCHÔA-FERNANDES, OLIVEIRA, I.; MOLINA, R.M.S., ZUCCHI, R.A. Species diversity of frugivorous flies (Díptera: Tephritoidea) from hosts in the Cerrado of the state of Mato Grosso do Sul, Brazil. Neotropical entomology, Londrina, v. 31, n. 4, p. 515-524, 2002.

VELOSO, V.R.S. Dinâmica populacional de Anastrepha spp. e Ceratitis capitata (Wied., 1824) (Diptera, Tephritidae) nos cerrados de Goiás. 1997. 115f. Tese (Doutorado em Ciências) - Escola de Agronomia, Universidade Federal de Goiás, Goiânia, 1997.

WHITE, I.M.; M.M. ELSON-HARRIS Fruit flies of economic significance: their identification and bionomics. Wallingford: CAB International, 1994.601p.

ZUCCHI, R.A. Espécies de Anastrepha, sinonímias, plantas hospedeiras e parasitóides. In: Malavasi, A. e Zucchi, R.A. (Ed.). Moscasdas-frutas de Importância Econômica no Brasil - conhecimento básico e aplicado. Ribeirão Preto: Holos Editora, 2000a. p. 41-48.

ZUCCHI, R.A. Mosca-do-mediterrâneo, Ceratitis capitata (Diptera: Tephritidae). In: VILELA, E., ZUCCHI, R.A.; CANTOR, F. (Ed.). Pragas introduzidas no Brasil. Ribeirão Preto: Holos Editora, 2000b. p. $15-22$. 